\title{
Guest editorial: fog computing on wheels
}

\author{
Hongzi Zhu ${ }^{1}$ - Tom H. Luan ${ }^{2}$ - Mianxiong Dong ${ }^{3} \cdot$ Peng Cheng ${ }^{4}$
}

Published online: 7 November 2017

(C) Springer Science+Business Media, LLC 2017

\section{Introduction}

Fog Computing is a paradigm that extends Cloud Computing, which emphasizes proximity to end-users and client objectives, dense geographical distribution and local resource pooling, latency reduction for quality of service, and edge analytics, resulting in superior user experience and redundancy in case of failure.

As more and more resources, such as computational power, storage, networking, and even electrical power, are integrated in vehicles, connected vehicles and roadside unites constitute a perfect Fog Computing platform. With the vast number of

This article is part of the Topical Collection: Special Issue on Fog Computing on Wheels

Guest Editors: Hongzi Zhu, Tom H. Luan, Mianxiong Dong, and Peng Cheng

Hongzi Zhu

hongzi@sjtu.edu.cn

Tom H. Luan

tom.luan@deakin.edu.au

Mianxiong Dong

mx.dong@csse.muroran-it.ac.jp

Peng Cheng

pcheng@iipc.zju.edu.cn

1 Shanghai Jiao Tong University, 800 Dongchuan Road, Shanghai 200240, China

2 Deakin University, 221 Burwood Highway, Burwood, VIC 3125 , Australia

3 Muroran Institute of Technology, 27-1 Mizumoto-cho, Muroran, Hokkaido 050-8585, Japan

4 Zhejiang University, 866 Yuhangtang Road, Hangzhou, Zhejiang Province 310058, China vehicles and their extraordinary mobility and wide distribution, a vehicular fog is the ideal platform to deliver a rich menu of services in infotainment, safety, traffic support, and analytics. The realization of vehicular fogs involves research in several disciplines including sensor technology, vehicular technology, wireless communication technology, and various technologies in computer science such as software engineering, artificial intelligence, big data analytics, and others. The research in vehicular fogs is interdisciplinary surrounding IoTs applications. Besides, the concept of vehicular fogs is new, with growing interest from the research community.

The aim of this special issue is to provide an introduction to the burgeoning theme of fog computing on connected vehicles and to collect articles about ideas, concepts, models, technologies, approaches, methodologies, and practices of vehicular fogs. Following an open call for papers, 15 technical papers are reviewed by at least three reviewers for their technical merit, scope, and relevance to the scope of this special issue, and 7 of them are selected and cover various areas within this theme, ranging from vehicular fog computing architecture to security problem in the system.

The first article, by Yuanguo Bi on "Neighboring vehicleassisted fast handoff for vehicular fog communications" proposes a cross-layer and neighboring vehicle-aided fast handoff scheme to provide a satisfactory user experience of accessing Internet on mobile vehicles. The second article entitled "Fog computing enabling geographic routing for urban area vehicular network" by Ting Lu, Shan Chang, and Wei Li presents a position-based routing scheme for the inter-vehicle communication in city environments, leveraging vehicular fog computing to make the best utilization of the vehicular communication and computational resources. In "Connection is power: Near optimal advertisement infrastructure placement for vehicular fogs", Wanru Xu, Panlong Yang, and Lijing Jiang undertake a theoretical analysis on mobile advertisement infrastructure deployment 
problem and the interaction between this deployment and vehicular fogs. The fourth article by Kai Fan, Junxiong Wang, Xin Wang, Hui Li, and Yintang Yang on "Secure, efficient and revocable data sharing scheme for vehicular fogs" presents how to utilize vehicular fog platform to achieve data sharing in vehicular networks. In "Towards fast and lightweight spam account detection in mobile social networks through fog computing", Jiahao Zhang, Qiang Li, Xiaoqi Wang, Bo Feng, and Dong Guo present how to use vehicular fog to detect spam account in mobile social networks in a fast and lightweight way. The sixth article on "Efficient multi-tasks scheduling algorithm in mobile cloud computing with time constraints" by Tongxiang Wang, Xianglin Wei, Chaogang Tang, and Jianhua Fan investigates the mobilecloud-computing-assisted execution of multi-tasks scheduling problem in hybrid computing architecture. The last (but not least) paper on "A multi-vessels cooperation scheduling for networked maritime fog-ran architecture leveraging SDN" by Tingting Yang, ZhengQi Cui, Rui Wang, Jian Zhao, Zhou Su, and Ruilong Deng integrates SDN and fog computing into maritime wideband communications system so as to achieve the minimized delay of weighted uploading packets.

Collectively, these 7 papers illustrate the diverse range of issues, providing a detailed compilation of the diverse range of issue currently being investigated in the field of vehicular fog computing.

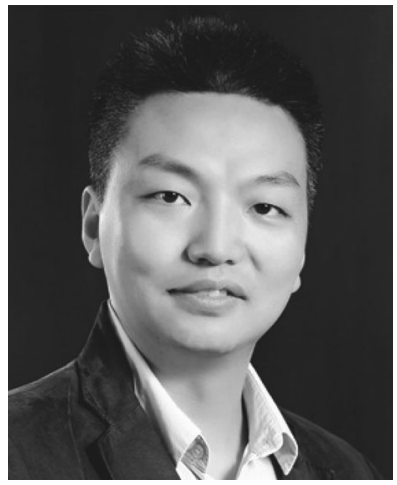

Hongzi Zhu received his B.S. and M.S. degrees at Jilin University in 2001 and 2004, respectively. He earned his Ph.D. degree in Computer Science from Shanghai Jiao Tong University in 2009. He was a Postdoctoral Fellow in the Department of Computer Science and Engineering at Hong Kong University of Science and Technology and the Department of Electrical and Computer Engineering at University of Waterloo in 2009 and 2010, respectively. He is now an associate professor at the Department of Computer Science and Engineering in Shanghai Jiao Tong University. His research interests include vehicular networks, mobile sensing and computing. He received the Best Paper Award from IEEE Globecom 2016. He is a member of the IEEE Computer Society and Communication Society.

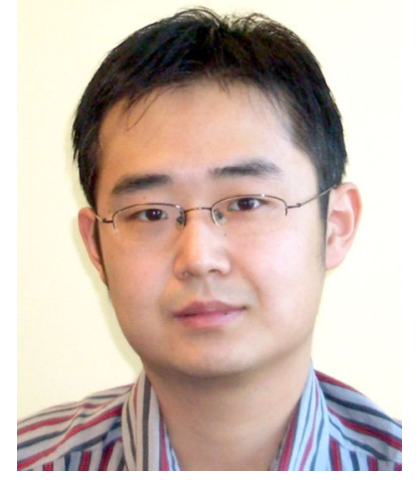

Tom H. Luan received his B.Eng. degree from Xi'an Jiao Tong University, China, in 2004, his M.Phil. degree from Hong Kong University of Science and Technology in 2007, and his $\mathrm{Ph} . \mathrm{D}$. degree from the University of Waterloo, Ontario, Canada, in 2012. He is currently with Xidian University at Xi'an of the People's Republic of China. He was a lecturer in the School of Information Technology at Deakin University, Melbourne, Australia, from 2014 to 2017. His research mainly focuses on content distribution and media streaming in vehicular ad hoc networks and peer-to-peer networking, as well as the protocol design and performance evaluation of wireless cloud computing and fog computing. Dr. Luan has authored/coauthored around 30 journal papers and 20 technical papers in conference proceedings, and awarded one US patent. He served as a TPC member for IEEE Globecom, ICC, PIMRC and the technical reviewer for multiple IEEE Transactions including TMC, TPDS, TVT, TWC and ITS.

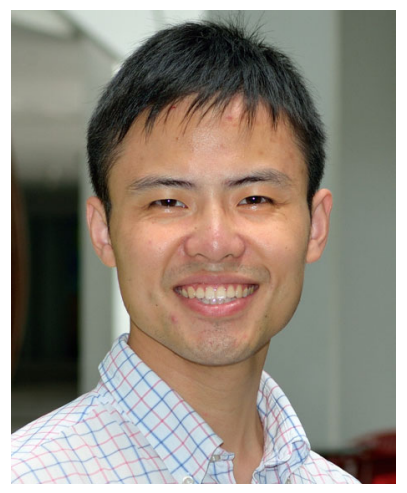

Peng Cheng received the B.E. degree in Automation, and the $\mathrm{Ph} . \mathrm{D}$. degree in Control Science and Engineering in 2004 and 2009 respectively, from Zhejiang University, Hangzhou, China. Currently he is Professor with College of Control Science and Engineering, Zhejiang University. He serves as Associate Editor of IEEE Transactions on Control of Network Systems, Wireless Networks, and International Journal of Communication Systems. He also serves/served as the Guest Editor for IEEE Transactions on Signal and Information Processing over Networks, and IEEE Transactions on Control of Network Systems. He served as the tpc co-chair of IEEE IOV 2016, local arrangement co-chair for ACM MobiHoc 2015, and the publicity cochair for IEEE MASS 2013. His research interests include networked sensing and control, cyber-physical systems, control system security. 


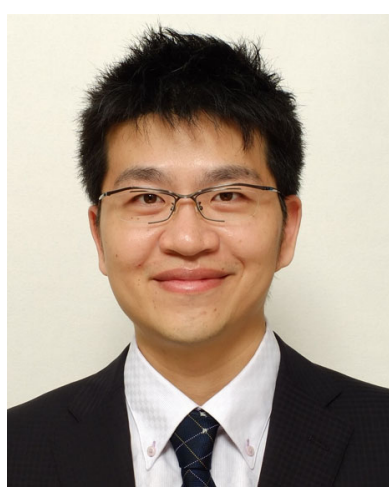

Mianxiong Dong received B.S., M.S. and Ph.D. in Computer Science and Engineering from The University of Aizu, Japan. $\mathrm{He}$ is currently an Associate Professor in the Department of Information and Electronic Engineering at the Muroran Institute of Technology, Japan. Prior to joining Muroran-IT, he was a Researcher at the National Institute of Information and Communications Technology (NICT), Japan. He was a JSPS Research Fellow with School of Computer Science and Engineering, The University of Aizu, Japan and was a visiting scholar with BBCR group at University of Waterloo, Canada supported by JSPS Excellent Young Researcher Overseas Visit Program from April 2010 to August 2011. Dr. Dong was selected as a Foreigner Research Fellow (a total of 3 recipients all over Japan) by NEC C\&C Foundation in 2011. Dr. Dong was a research scientist with A3 Foresight Program (2011-2016) funded by Japan Society for the Promotion of Sciences (JSPS), NSFC of China, and NRF of Korea. He is the recipient of IEEE TCSC Early Career Award 2016. 